Muro de la Investigación, 2020(1), enero-junio ISSN: 2523-2886

Doi: https://doi.org/10.17162/rmi.v5i1.1299

\title{
Bienestar espiritual y calidad de atención de los profesionales de enfermería en dos hospitales de Lima Metropolitana
}

\author{
Spiritual well-being and quality of care of nursing professionals in two hospitals in \\ Lima Metropolitan City \\ Stephanie Johana Valera Montalvan ${ }^{1 a}$, Lucero Marisol Romero Bueno ${ }^{2 a}$ y \\ Rut Ester Mamani Limachi ${ }^{3}$ \\ Universidad Peruana Unión, Perú ${ }^{123}$
}

Recibido: 07 de octubre de 2019

Aceptado: 12 de diciembre de 2019

\begin{abstract}
Resumen
El presente estudio determina la relación entre el nivel de bienestar espiritual y la calidad de atención de los profesionales de enfermería en dos hospitales de Lima Metropolitana. Se emplea un enfoque cuantitativo, de diseño no experimental, correlacional y de corte transversal. La selección de la muestra estuvo compuesta por 60 profesionales de enfermería y 180 pacientes. Los instrumentos utilizados para la recolección de datos fueron: el Cuestionario de "Escala de bienestar espiritual" (Ellison y Paulotzian, 1994) y el Cuestionario de "Escala de calidad de atención" (MINSA y Colegio de Enfermeros del Perú, 2008). Los resultados encontrados indicaron que el 53.3\% del personal de enfermería presentó un nivel de bienestar espiritual medio y solo el $5.0 \%$ un nivel alto; asimismo, el 56,7\% presenta un nivel de calidad de atención bajo y solo el $8.3 \%$ un nivel alto. Además, se encontró que existe relación entre el bienestar espiritual y la calidad de atención de los profesionales de enfermería con un coeficiente de 0,540 y un p-valor de 0,000 a través de la prueba Tau b de Kendall. En conclusión, sí existe relación entre ambas variables de estudio.
\end{abstract}

Palabras Clave: Bienestar espiritual, calidad de atención, profesionales de enfermería, salud

\begin{abstract}
This study determines the relationship between the level of spiritual well-being and the quality of care of nursing professionals in two hospitals in Lima Metropolitan City. A quantitative, non-experimental, correlational, and cross-sectional approach is used. The sample selection was made up of 60 nursing professionals and 180 patients. The instruments used for data collection were: the "Scale of spiritual well-being" Questionnaire (Ellison and Paulotzian, 1994) and the Questionnaire of "Scale of quality of care" (MINSA and the College of Nurses of Peru, 2008). The results found indicated that $53.3 \%$ of the nursing staff presented a medium level of spiritual well-being and only
\end{abstract}

${ }^{\mathrm{a}}$ Correspondencia a las autoras

E-mail: stephanievalera@upeu.edu.pe; luceroromero@upeu.edu.pe 
$5.0 \%$ a high level; Likewise, $56.7 \%$ present a low level of quality of care and only $8.3 \%$ a high level. Furthermore, it was found that there is a relationship between the spiritual well-being and the quality of care of nursing professionals with a coefficient of 0.540 and a p-value of 0.000 through the Kendall Tau b test. In conclusion, there is a relationship between both study variables.

Keywords: Spiritual well-being, quality of care, nursing, health

\section{Introducción}

Según la Organización Mundial de la Salud (OMS), la calidad de la asistencia sanitaria busca que cada paciente reciba el conjunto de servicios adecuados para una atención sanitaria óptima y lograr la máxima satisfacción del paciente en el proceso (Ortiz, 2014). Por su parte, Castillo (2011) indica que la calidad de atención en salud es hoy día un objetivo fundamental y Sierra y Montalvo (2012) afirman que la espiritualidad en enfermería ha tenido especial relevancia con el transcurrir del tiempo, desde el inicio de la teoría de Nightingale, en 1859, se creyó en el componente espiritual de la vida humana.

Un estudio realizado en Brasil, titulado "Calidad de los cuidados de enfermería y satisfacción del paciente atendido en un hospital de Brasil”, investigó la calidad de los cuidados de enfermería y su relación con la satisfacción del paciente. Se llega a la conclusión que la enfermería tiene dificultad al ampliar el alcance de sus cuidados, e incluir las necesidades emocionales y psico-espirituales de los pacientes. (Santana et al., 2014).

Así también, Mesquita et al. (2014) comprobaron que el22\% afirmó nunca prestar el cuidado espiritual, $46 \%$ raramente, $30 \%$ frecuentemente y $2 \%$ no respondió. Sin embargo, el 95\% de los sujetos consideraron importante la prestación del cuidado espiritual; el 78\% consideran importante que los profesionales de salud aborden la espiritualidad y la religiosidad a sus pacientes y el $42 \%$ afirma no haber recibido formación al respecto. Esto indica la importancia de la espiritualidad en la vida de los profesionales, sea en relación a su vivencia personal o a su labor profesional, y la importancia de la misma en el cuidado al paciente (Mesquita et al., 2014).

En el Perú, se halló que el $60 \%$ de los pacientes tuvieron una percepción medianamente favorable sobre la atención de enfermería y solo un $40 \%$ de los pacientes señalaron que la atención fue favorable. (Silva et al., 2015). Por otro lado, el Ministerio de Salud (MINSA, 2009) afirma que la población percibe baja calidad en la atención que recibe y también una tendencia a seguir descendiendo, tanto para los hospitales del 
MINSA, y de EsSalud. Esta inadecuada situación puede encontrar una explicación en la insuficiente voluntad política de la autoridad sanitaria para remediar este problema.

En este contexto, la presente investigación busca determinar la relación entre el nivel de bienestar espiritual y la calidad de atención de los profesionales de enfermería en dos hospitales de Lima Metropolitana. Esto será de beneficio para los profesionales de la salud - en especial de enfermería - en el incentivo y énfasis de la necesidad del aspecto espiritual en sus propias vidas, y así brindar una atención de calidad hacia sus pacientes.

\section{Metodología}

Esta investigación presenta un enfoque cuantitativo, no experimental, de corte transversal y de tipo correlacional. La muestra estuvo conformada por 60 profesionales de enfermería y 180 pacientes que fueron elegidos de manera no probabilística y convencional. El "Cuestionario de bienestar espiritual" fue tomado de Ellison y Paulotzian (1994) y consta de dos partes: la primera con 10 preguntas referentes al bienestar existencial y la segunda con 10 preguntas referente al bienestar existencial, lo cual se adaptó del instrumento de bienestar espiritual diseñado por Flores y Flores (2015). El método que se utilizó para comprobar la fiabilidad de dicho instrumento fue el alfa de Cronbach, obteniendo un valor de 0,89 en la variable bienestar espiritual; 0,87 en la dimensión de bienestar religioso y 0,78 en la dimensión de bienestar existencial.

Así también, el instrumento de calidad de atención fue elaborado en base a las dimensiones consideradas en las normas de gestión de calidad del MINSA y del Colegio de Enfermeros del Perú (CEP), y consta de seis partes: 18 preguntas para la dimensión de técnico-científico (eficacia, continuidad, seguridad e integridad) y 12 preguntas de la dimensión entorno y humana. La fiabilidad de dicho instrumento se comprobó mediante el Alfa de Crombach obtenido un valor de 0.803 .

\section{Resultados}

Tabla 1

Nivel del bienestar espiritual de los profesionales de enfermería en dos hospitales de Lima Metropolitana, 2019

\begin{tabular}{lll}
\hline Variable & $\mathrm{N}$ & $\%$ \\
\hline Bienestar espiritual & & \\
Bajo & 25 & 41.7
\end{tabular}


Bienestar religioso

Bajo

Medio

Alto

Bienestar existential

$$
\text { Bajo }
$$

Medio

En la tabla 1 se observa que del $100 \%$ de los profesionales de enfermería, el $53.3 \%$ presentan un nivel de bienestar espiritual medio y solo el 5.0\% un nivel alto. Con respecto al bienestar religioso, el 60.0\% tiene un nivel bajo y solo el 3,3\% un nivel alto. En cuanto al bienestar existencial, el $65.0 \%$ tiene un nivel medio, mientras que el $8.3 \%$ un nivel alto.

\section{Tabla 2}

Nivel de la calidad de atención de los profesionales de enfermería en dos Hospitales de Lima Metropolitana, 2019

\begin{tabular}{lcc}
\hline Calidad de la atención & $\mathrm{N}$ & $\%$ \\
\hline Bajo & 34 & 56.7 \\
Medio & 21 & 35.0 \\
Alto & 5 & 8.3 \\
Total & 60 & 100.0 \\
\hline
\end{tabular}

En la tabla 2 se observa que del $100 \%$ de los profesionales de enfermería, el 56,7\% presentan un nivel de calidad de atención bajo y solo el $8.3 \%$ un nivel alto. 


\section{Tabla 3}

Correlación entre el bienestar espiritual y la calidad de atención de los profesionales de enfermería en dos Hospitales de Lima Metropolitana, 2019

\begin{tabular}{lcccccc}
\hline & & \multicolumn{3}{c}{ Calidad de atención } & \multicolumn{2}{c}{ Total } \\
\cline { 3 - 6 } & & Bajo & Regular & Alto & To \\
\hline Bienestar & Bajo & $\mathrm{n}$ & 22 & 3 & 0 & 25 \\
& & $\%$ & $36,7 \%$ & $5,0 \%$ & $0,0 \%$ & 41.7 \\
& Medio & $\mathrm{n}$ & 12 & 16 & 4 & 32 \\
& & $\%$ & $20,0 \%$ & $26,7 \%$ & $6,7 \%$ & 53.3 \\
& Alto & $\mathrm{n}$ & 0 & 2 & 1 & 3 \\
& & $\%$ & $0,0 \%$ & $3,3 \%$ & $1,7 \%$ & 5 \\
& Total & $\mathrm{n}$ & 34 & 21 & 5 & 60 \\
& $\%$ & 56.7 & 35 & 8.3 & 100 \\
\hline
\end{tabular}

En la tabla 3 se observa que del $100 \%$ de la muestra, el $36.7 \%$ de los profesiona les de enfermería tienen un nivel de bienestar espiritual bajo y una calidad de atención baja. Asimismo, el $1.7 \%$ de los participantes tienen un nivel de bienestar espiritual alto y una calidad de atención alta. Para determinar la relación entre ambas variables se empleó la prueba estadística Tau-b de Kendall, donde se obtuvo un p-valor de .000 y un coeficiente de correlación moderado positivo de .540 , lo que indica que existe relación entre el bienestar espiritual y la calidad de atención de los profesionales de enfermería en dos Hospitales de Lima Metropolitana.

\section{Tabla 4}

Correlación entre el bienestar religioso y la calidad de atención de las profesionales de enfermería en dos Hospitales de Lima Metropolitana, 2019

\begin{tabular}{llllllll}
\hline & & \multicolumn{5}{c}{ Calidad de atención } & \\
\cline { 3 - 5 } & & & Bajo & Regular & & Alto & Total \\
\hline Bienestar & Bajo & $\mathrm{N}$ & 33 & 3 & 0 & 36 \\
religioso & & $\%$ & $55,0 \%$ & $5,0 \%$ & $0,0 \%$ & 60 \\
& Medio & $\mathrm{N}$ & 1 & 18 & 3 & 22 \\
& & $\%$ & $1,7 \%$ & $30,0 \%$ & $5,0 \%$ & 36.7 \\
& Alto & $\mathrm{N}$ & 0 & 0 & 2 & 2 \\
\hline
\end{tabular}




\begin{tabular}{llllll}
\hline & $\%$ & $0,0 \%$ & $0,0 \%$ & $3,3 \%$ & 3.3 \\
Total & $\mathrm{N}$ & 34 & 21 & 5 & 60 \\
& $\%$ & 56.7 & 35 & 8.3 & 100 \\
\hline
\end{tabular}

En la tabla 4 se observa que del $100 \%$ de los profesionales de enfermería, el $55.0 \%$ de los enfermeros tienen un nivel de bienestar religioso bajo y una calidad de atención baja. Asimismo, el 1.7\% de los participantes tienen un nivel de bienestar religioso medio y una calidad de atención baja. Para determinar, la relación entre ambas variables se empleó la prueba estadística Tau-b de Kendall, donde se obtuvo un p-valor de .000 y un coeficiente de correlación moderado positivo de .854 , lo que indica que existe relación entre el bienestar religioso y la calidad de atención de los profesionales de enfermería en dos hospitales de Lima Metropolitana.

\section{Tabla 5}

Correlación entre el bienestar existencial y la calidad de atención de los profesionales de enfermería en dos Hospitales de Lima Metropolitana, 2019.

\begin{tabular}{|c|c|c|c|c|c|c|}
\hline & & & \multicolumn{3}{|c|}{ Calidad de Atención } & \multirow[b]{2}{*}{ Total } \\
\hline & & & Bajo & Regular & Alto & \\
\hline \multirow{6}{*}{$\begin{array}{l}\text { Bienestar } \\
\text { existencial }\end{array}$} & Bajo & $\mathrm{n}$ & 16 & 0 & 0 & 16 \\
\hline & & $\%$ & $26,7 \%$ & $0,0 \%$ & $0,0 \%$ & 26.7 \\
\hline & Medio & $\mathrm{n}$ & 18 & 20 & 1 & 39 \\
\hline & & $\%$ & $30,0 \%$ & $33,3 \%$ & $1,7 \%$ & 65 \\
\hline & Alto & $\mathrm{n}$ & 0 & 1 & 4 & 5 \\
\hline & & $\%$ & $0,0 \%$ & $1,7 \%$ & $6,7 \%$ & 8.3 \\
\hline \multirow[t]{2}{*}{ Total } & & $\mathrm{n}$ & 34 & 21 & 5 & 60 \\
\hline & & $\%$ & 56.7 & 35 & 8.3 & 100 \\
\hline
\end{tabular}

En cuanto a la relación entre bienestar existencial y calidad de la atención, en la Tabla 5, se observa que todos los que se encontraron en un nivel bajo de bienestar existencial, también ofrecían una baja calidad de atención. Asimismo, la mayoría $(33,3 \%)$ tenían un nivel medio de bienestar existencial y ofrecían una regular calidad de atención. También se encontró que casi todos los que tenían un alto bienestar existencial tenían en su totalidad una alta calidad de atención. La estrecha y significativa relación que se ha 
encontrado entre el bienestar existencial y la calidad de la atención, se demuestra por el valor Tau b de Kendall $(0,620)$ que se ha hallado.

\section{Discusión}

El 53.3\% presenta un nivel de bienestar espiritual medio y solo el 5.0\% un nivel de bienestar espiritual alto (ver tabla 1). Resultados similares encontraron Mesquita et al. (2014), donde el 96\% de los profesionales de enfermería en Brasil presentaron un nivel de bienestar espiritual moderado y un $4 \%$ un nivel de bienestar espiritual alto. De igual manera, Guerrero, Meneses y De la Cruz (2015) evidenciaron que el 87\% presentó un nivel de bienestar espiritual regular y solo un $4 \%$ bajo y un $9 \%$ alto.

Según Watson (2014), las enfermeras que posean una baja espiritualidad no podrán tener comportamientos adecuados en el cuidado del paciente. Para McSherry et al. (2013, citados por Vargas, 2015), esto adquiere dos formas: una religiosa y otra existencial. La primera se deriva de una tradición histórica y se basa sobre descriptores religiosos y teocéntricos.

En cuanto a la calidad de atención, los resultados evidencian que el 56,7\% presenta un nivel de calidad de atención bajo, $41,1 \%$ un nivel regular de calidad de atención y solo el 2,2\% un nivel alto de calidad (ver tabla 2); resultados similares encontró Díaz (2014), que obtuvo como resultado que el 68,2\% se encuentra insatisfecho de acuerdo a la calidad de atención brindada y el 32,8\% está satisfecho. Del mismo modo, Silva et al. (2015) concluyeron que el $60 \%$ de los pacientes tuvieron una percepción medianamente favorable y el $40 \%$ no favorable.

Por su parte, Vizcarra (2013) indica que la baja calidad de atención de las enfermeras es debido a que se encuentra en permanente interacción con factores generadores de estrés, uno de ellos es la sobrecarga laboral, así mismo las condiciones de trabajo (inadecuado ambiente físico laboral); estos son riesgos para la seguridad y salud del trabajador. Asimismo, Espinoza, Luengo y Sanhueza (2016) señalan otro factor importante para la inadecuada atención, son las intensas reacciones emocionales, que, de no ser atendidas y canalizadas de forma apropiada, terminan en manifestaciones de ansiedad y estrés.

En otro orden, Morales (2004) señala que, en cuanto al turno y a la edad del personal de enfermería, las enfermeras jóvenes difieren en su desempeño con las enfermeras de mayor edad, lo que puede contribuir a que las primeras lleguen con menor cansancio y sean más tolerantes y se involucren más en la atención. Además, Aguirre 
(2009) refiere que las enfermeras que trabajan cuidando a los pacientes críticos, están más satisfechas laboralmente que las demás enfermeras que laboran en otros servicios.

En otro aspecto, Novoa et al. (2004) refieren que, al no existir una buena comunicación entre las jefas de enfermería y las auxiliares, se generan altos niveles de estrés que afecta al mismo personal y a los pacientes, sobre todo en las horas de la mañana y de la tarde que son las que experimentan mayor afluencia de familiares, estudiantes y médicos. Asimismo, siendo que el profesional de enfermería-además de cumplir funciones profesionales y familiares-debe cumplir otras funciones de cuidado del paciente (ser madre, esposa, hija, hermana), se halla expuesto a factores estresantes, y por ello, se podría generar una mala calidad de atención hacia al paciente (Velásquez, 2016).

En torno a la hipótesis de estudio, se demostró que a mayor nivel de bienestar espiritual mejora la calidad de atención de los profesionales de atención con un p-valor de p<.000 (ver tabla 3). Resultado similar obtuvo García (2018) en su estudio titulado "Estrés, cuidado espiritual y percepción de los comportamientos de cuidado del personal de enfermería", donde encontró que existe correlación entre el cuidado espiritual y la percepción de los comportamientos del cuidado $(\mathrm{p}<.05)$. De manera semejante, Melgar y Estuco (2014) encontraron que existe relación significativa entre la espiritualidad y la calidad del servicio según la percepción de los pacientes $(\mathrm{p}<.05)$.

Del mismo modo, en cuanto al análisis del nivel de bienestar espiritual y la calidad de atención de los profesionales de enfermería (ver Tabla 3), Sierra y Montalvo (2012) reafirman que la enfermera, además de su labor de cuidado integral al paciente, tiene la obligación de no olvidar la dimensión espiritual de la persona. La evaluación espiritual en sí misma es terapéutica, pues los pacientes con enfermedades en etapa terminal la viven con una preocupación e interés por parte del profesional. Consecuentemente, Mesquita et al. (2014), resalta que la espiritualidad como una dimensión relevante en la vida de los profesionales, sea en relación a su vivencia personal o a la presencia de aquella en su labor profesional; necesitan de este soporte para que la prestación del cuidado sea más humanizada. En este sentido, García (2018) refiere que el cuidado espiritual de la enfermera se convierte en un factor determinante al momento de brindar cuidado, demostrando los comportamientos más cálidos de amor y compasión que se puedan brindar. Con la presencia de la enfermera se cultiva su propio ser espiritual haciendo auténtica labor humanitaria y de servicio, con calidad en la atención del paciente. Por lo dicho anteriormente, Ruder (citado por Mesquita et al., 2014), recalca que es vital que los profesionales de enfermería respondan a las necesidades espirituales de los pacientes de 
una forma competente $\mathrm{y}$ sensible, por eso es importante que los profesionales de enfermería también exploren su propia espiritualidad y la relación de esta con el cuidado, pues eso puede generar un impacto importante sobre la conciencia y la sensibilidad de las necesidades espirituales de los pacientes. En conclusión, se confirma que sí existe correlación entre el bienestar espiritual, religioso y existencial con la calidad de atención de los profesionales de enfermería en dos hospitales de Lima Metropolitana.

\section{Referencias}

Aguirre, D. (2009). Satisfacción laboral de los recursos humanos de enfermería.

Factores que la afectan. Revista Habanera de Ciencias Médicas, 8(4), 102-106.

Recuperado de https $/ / w w w . r e d a l y c . o r g / p d f / 1804 / 180414045021 . p d f$

Castillo, G. (2011). Grado de satisfacción del usuario externo con la calidad de atención en la consulta externa que se desarrolla en el Centro Médico Esmil.

(Título de Diploma Superior en Gerencia y Calidad en Servicios de Salud).

Universidad Católica de la Loja. Recuperado de:

http://dspace.utpl.edu.ec/bitstream/123456789/2618/3/UTPL_Castillo_Gladys_36 0X1573.pdf

Díaz, M. (2014). Satisfacción del usuario externo sobre la calidad de atención de salud, Hospital Tito Villar Cabeza, Bambamarca (Tesis de pregrado). Universidad Nacional de Cajamarca, Perú. Recuperado de:

http://repositorio.unc.edu.pe/bitstream/handle/UNC/143/T\%20610.73\%20D542\% 202014.pdf? sequence $=1 \&$ is Allowed $=y$

Espinoza, M., Luengo, L. y Sanhueza, O. (2016). Actitudes en profesionales de enfermería chilenos hacia el cuidado al final de la vida. Análisis multivariado. Revista Aquichan, 16(4), 430-446. Recuperado de http://www.scielo.org.co/pdf/aqui/v16n4/1657-5997-aqui-16-04-00430.pdf

García, M. (2018). Estrés, cuidado espiritual y percepción de los comportamientos de cuidado del personal de enfermería (Tesis de posgrado). Universidad Nacional de Colombia. Recuperado de http//bdigital.unal.edu.co/70245/1/ 52729907.2018.pdf

Guerrero, R., Meneses, M. y De la Cruz-Ruiz, M. (2015). Cuidado humanizado de enfermería según la teoría de Jean Watson, servicio de medicina del Hospital Daniel Alcides Carrión, Lima, Callao, 2015. Revista de Enfermería Herediana, 
9(12), 133-142. Recuperado de http://repositorio.ucv.edu.pe/ bitstream/handle/UCV/19486/3017-7642-1-PB.pdf? sequence=1\&isAllowed=y

Melgar, M. y Estuco, J. (2014). Relación de espiritualidad y escala de valores de la enfermera con la percepción del paciente en el servicio de emergencia del hospital Honorio Delgado. Arequipa - 2014 (Tesis de segunda especialidad). Universidad Nacional De San Agustín. Recuperado de:

http://repositorio.unsa.edu.pe/bitstream/handle/UNSA/3185/ENS mealmj.pdf?sequ ence $=1 \&$ is Allowed $=\mathrm{y}$

Mesquita, A., Costa, C., Neves, M., Alves, D., Souza, F. y López, E. (2014). El bienestar espiritual y la prestación del cuidado espiritual en un equipo de enfermería. Revista Index de Enfermería, 23(4), 219-223. Recuperado de http://www.indexf.com/index-enfermeria/v23n4/pdf/9471.pdf

Ministerio de Salud (2009). Documento Técnico: Política Nacional de Calidad en Salud. Recuperado de http://bvs.minsa.gob.pe/local/minsa/1997.pdf

Morales, M. (2004). Calidad de atención desde la perspectiva del paciente (Tesis de maestría). Universidad Autónoma de Nuevo León, México. Recuperado de: http://eprints.uanl.mx/6416/1/1080111072.PDF

Novoa, M., Nieto, C., Forero, C., Caycedo, C., Palma, M., Montealegre, M, Bayona M. y Sánchez, C. (2004). Relación entre perfil psicológico, calidad de vida y estrés asistencial en personal de enfermería. Revista de la Pontifica Universidad Javeriana, $\quad 4(1), \quad 63-75 . \quad$ Recuperado de http://www.scielo.org.co/pdf/rups/v4n1/v4n1 a09.pdf

Ortiz, P. (2014). Nivel de satisfacción del usuario externo sobre la calidad de atención en los servicios de salud según encuesta Servqual, en la micro red VillaChorrillos en el año 2014 (Tesis de pregrado). Universidad Ricardo Palma, Peru. Recuperado de: http://repositorio.urp.edu.pe/bitstream/handle/urp/457/Ortiz_p.pdf? sequence=1\&is Allowed $=\mathrm{y}$

Santana, J., Bauer, A. E., Minamisava, R., Queiroz, A. y Gomes, M. (2014). Calidad de los cuidados de enfermería y satisfacción del paciente atendido en un hospital de enseñanza. Revista Latino Americano Enfermagem, 22(3), 454-460. Recuperado de http:/www.scielo.br/pdf/rlae/v, 22n3/es_0104-1169-rlae-22-03-00454 
Sierra, L. y Montalvo, P. (2012). Bienestar espiritual de enfermeras y enfermeros en unidades de cuidado intensivo. Revista avances en Enfermería, 30(1), 64-74. Recuperado de http://www.index-f.com/rae/301pdf/064074.pdf

Silva, J., Ramón, S., Vergaray, S., Palacios, V. y Partezani, R. (2015). Percepción del paciente hospitalizado respecto a la atención de enfermería en un hospital público. Revista de enfermería universitaria, 12(2), 80-87. Recuperado de http://www.scielo.org.mx/pdf/eu/v12n2/1665-7063-eu-12-02-00080.pdf

Velásquez, C. (2016). Calidad de vida profesional que tienen las enfermeras que laboran en el Hospital San José de Chincha (Tesis de pregrado). Universidad San Juan Bautista, Perú. Recuperado de http://repositorio.upsjb.edu.pe/bitstream/handle/upsjb/530/T-TPLE-Ceferina $\% 20$ Velasquez\%20Ceperian.pdf? sequence $=1 \&$ isAllowed $=\mathrm{y}$

Vargas, L. (2015). Evaluación de una intervención educativa para fortalecer el cuidado espiritual en el personal de enfermería que trabaja con personas con enfermedad crónica (Tesis de posgrado). Universidad Nacional de Colombia. Recuperado de http://bdigital.unal.edu.co/49010/1/5539734.2015.pdf

Vizcarra, J. (2013). Estrés laboral y su relación con la calidad de cuidado que brinda el profesional de enfermería en la Micro Red de Salud, Cono Sur, Tacna (Tesis de pregrado). Universidad Nacional Joreg Basadre Grohmann, Perú. Recuperado de http://repositorio.unjbg.edu.pe/bitstream/handle/UNJBG/2293/80_2013_vizcarra_ villanueva_jc_facs_enfermeria.pdf?sequence $=1 \&$ isAllowed $=\mathrm{y}$ 